\title{
Personalizing Medication Recommendation with a Graph-Based Approach
}

\author{
SUMAN BHOI, MONG LI LEE, and WYNNE HSU, National University of Singapore \\ HAO SEN ANDREW FANG and NGIAP CHUAN TAN, SingHealth Polyclinics
}

\begin{abstract}
The broad adoption of electronic health records (EHRs) has led to vast amounts of data being accumulated on a patient's history, diagnosis, prescriptions, and lab tests. Advances in recommender technologies have the potential to utilize this information to help doctors personalize the prescribed medications. However, existing medication recommendation systems have yet to make use of all these information sources in a seamless manner, and they do not provide a justification on why a particular medication is recommended. In this work, we design a two-stage personalized medication recommender system called PREMIER that incorporates information from the EHR. We utilize the various weights in the system to compute the contributions from the information sources for the recommended medications. Our system models the drug interaction from an external drug database and the drug co-occurrence from the EHR as graphs. Experiment results on MIMIC-III and a proprietary outpatient dataset show that PREMIER outperforms state-of-the-art medication recommendation systems while achieving the best tradeoff between accuracy and drug-drug interaction. Case studies demonstrate that the justifications provided by PREMIER are appropriate and aligned to clinical practices.
\end{abstract}

\section{CCS Concepts: • Information systems $\rightarrow$ Recommender systems;}

Additional Key Words and Phrases: Electronic health records, attention-based recommender system

\section{ACM Reference format:}

Suman Bhoi, Mong Li Lee, Wynne Hsu, Hao Sen Andrew Fang, and Ngiap Chuan Tan. 2021. Personalizing Medication Recommendation with a Graph-Based Approach. ACM Trans. Inf. Syst. 40, 3, Article 55 (November 2021), 23 pages.

https://doi.org/10.1145/3488668

\section{INTRODUCTION}

Technological advances and digitization have led to the increasing availability of patient information for healthcare data analytics. The large repositories of electronic health records (EHRs) contain comprehensive information about patient visits over time, procedures, lab tests results, disease diagnosis, prescribed medications, and so on. Research on EHRs has ranged from disease inference [26] and outcome prediction [14,24] to readmission and mortality estimation [25, 38].

This research was supported by the National Research Foundation, Singapore, under its AI Singapore Programme (AISG Award No. AISG-GC-2019-001)

Authors' addresses: S. Bhoi, National University of Singapore, Singapore; email: sumanbhoi@u.nus.edu; M. L. Lee, National University of Singapore, Singapore; email: leeml@comp.nus.edu.sg; W. Hsu, National University of Singapore, Singapore; email: whsu@comp.nus.edu.sg; H. Sen Andrew Fang, SingHealth Polyclinics, Singapore; email: andrew.fang.h.s@singhealth.com.sg; N. C. Tan, SingHealth Polyclinics, Singapore; email: tan.ngiap.chuan@singhealth. com.sg.

Permission to make digital or hard copies of all or part of this work for personal or classroom use is granted without fee provided that copies are not made or distributed for profit or commercial advantage and that copies bear this notice and the full citation on the first page. Copyrights for components of this work owned by others than the author(s) must be honored. Abstracting with credit is permitted. To copy otherwise, or republish, to post on servers or to redistribute to lists, requires prior specific permission and/or a fee. Request permissions from permissions@acm.org.

(C) 2021 Copyright held by the owner/author(s). Publication rights licensed to ACM.

1046-8188/2021/11-ART55 \$15.00

https://doi.org/10.1145/3488668 
The White House Precision Medicine Initiative ${ }^{1}$ has propelled the move to develop and personalize treatment regimens to improve health outcomes.

The traditional "one size fits all" concept is being superseded by treatments that are tailor-made for each individual. One line of research in this direction is the personalization of medication recommender systems. In the largest publicly available benchmark dataset MIMIC-III [16], a patient has an average of 20 medications. Eliminating all the potential drug-to-drug interactions remains a challenge. Although a clinician tries to ensure that the medications prescribed do not have the common drug-to-drug interactions, having a medication recommender system suggest a list of medicines given a patient's diagnosis, test procedures, and so on that minimizes adverse drug reactions will serve as an important decision support tool to the clinician.

Besides recommending a list of medications, providing justifications for these recommendations is crucial to build trust, thereby increasing clinicians' acceptance of the system and patients' adherence to the medications. Hence, an ideal personalized recommender system should (a) incorporate patient-specific information, such as diagnosis and procedures underwent, culminated over time to personalize the recommendations; (b) incorporate drug interaction information to minimize adverse drug interaction; and (c) provide justification for the recommendations rendered.

Existing recommender systems such as LEAP [47] use the records of patients' current visits and the drug-to-drug interactions to predict a list of medications and do not consider information from patients' previous visits, leading to lower accuracy. Other recommender systems such as DMNC [21] capture the temporal dependencies in patients' past visits when predicting medications but do not consider the interactions between drugs that may lead to adverse drug events, particularly in patients with co-morbidity [27]. For example, the usage of anti-inflammatory and anti-rheumatic medications together with sedatives could result in diabetic retinopathy [40].

Graph neural networks have been gaining traction for their ability to model dependencies in graph data. Popular graph neural network models include the graph convolutional network (GCN) [19], GraphSAGE [13], and graph attention network (GAT) [42]. GameNet [30] employs dynamic memory in conjunction with recurrent neural networks (RNNs) to model temporal dependency in patients' EHRs and uses GCN [19] to model the interaction between drugs. CompNet [45] is a medication recommender system that is based on reinforcement learning. It utilizes relationalGCN (R-GCN) [28] to capture the drug interactions. All these approaches do not use a weighted combination of a patient's diagnosis, procedures, and past medications when retrieving relevant drug interaction information, and are unable to provide justifications for the recommendations.

Medication recommender systems that use GCN to model drug interaction information are unable to capture the severity of different drug interactions. This is because GCN uses a version of the convolutional neural network that operates on a fixed-size neighborhood of nodes in graphs and cannot model varying contributions of nodes. This limitation may have serious implications in practice. For example, the adverse drug event of long-term or permanent paralysis as a result of using an anti-inflammatory medication like ibuprofen together with an anti-coagulant medication like enoxaparin is clearly more severe compared to having diarrhea after using ibuprofen and a constipation medication like linaclotide. Motivated by the work of Veličkovič et al. [42] which proposed GAT to learn the varying importance of nodes in the same neighborhood by using multihead attention, we use GATs to deal with the co-occurrence and interaction information between different drugs.

In this work, we propose a recommender system for medications called PREMIER that takes into account the past and current visits of patient records, drug co-occurrences in the EHR, and the known interactions of drugs, to generate personalized medications with justifications while

\footnotetext{
${ }^{1}$ https://obamawhitehouse.archives.gov/precision-medicine.
} 
minimizing adverse drug reactions. There are two key stages in PREMIER. The first stage utilizes a two-level neural attention model instead of the traditional RNN to extract patient-specific information such as the patient's diagnosis, procedures, and his/her previously prescribed medications to create a personalized query vector. The second stage uses this query vector to retrieve information about potential drug interactions and drug co-occurrences from the GAT-based graphical representations of the drug interaction repository and EHR, respectively. With this, PREMIER ensures that the predicted set of medications has minimal drug interactions and is able to identify the top contributors for each medication recommended, thereby giving the justifications for the recommendations.

Extensive quantitative and qualitative experiments on the benchmark MIMIC-III EHR and a proprietary outpatient dataset demonstrate that PREMIER outperforms state-of-the-art medication recommender systems in terms of accuracy with lower DDI. We also show that PREMIER is able to recommend medications for patients diagnosed with rare diseases despite the limited data. Case studies and clinician assessment indicate that the justifications provided by PREMIER are aligned with clinical practices. We summarize our contributions as follows:

- We propose a two-stage recommender system comprising attention-based RNNs to model patient visits and graph networks to model drug co-occurrences in the EHR and known drug interactions.

- We adapt GAT to incorporate the varying importance of drug interactions to learn effective drug embeddings for the task of medication recommendation.

- We justify the key reasons for recommending a particular medication by providing the percentage of contributions among the diagnosis, procedures, and previously prescribed medications.

- We present extensive analysis and assessment on two real-world datasets.

\section{RELATED WORK}

Research in medication recommender systems can be broadly classified into rule-based, instancebased, and longitudinal systems.

Rule-based recommendation. This approach typically relies on a collection of rules that capture the knowledge of a medical expert. Solt and Tikk [35] propose rules to extract medication information from discharge summaries. Chen et al. [4] derive knowledge patterns from medical information such as disease, symptoms, demographics, and measurements of a patient to recommend preferred treatments for chronic heart failure.

Gunlicks-Stoessel et al. [12] attempt to develop adaptive treatment recommendation for adolescent depression based on hard-coded protocols. Lakkaraju and Rudin [20] use a Markov decision process (MDP) to learn the mapping between patient characteristics and treatments. In general, rule-based systems cannot scale or generalize well. Further, it is difficult to add rules to an already large knowledge base without introducing conflicting rules.

Instance-based recommendation. In this approach, only the current visits of patients are used for medication recommendation. Zhang et al. [47] formulate the medication recommender problem as a multi-instance multi-label learning task and develop the LEAP algorithm. A recurrent decoder is used to facilitate sequential decision making to model the drug-disease relation along with DDI from the EHRs. Once this model is trained on patients' EHRs, it predicts medications based on the diagnosis of a patient's current visit.

Wang et al. [44] jointly embed patient demographics, diagnosis, and past medications into a lower-dimensional space and use this embedding for recommendation. They formulate the task of drug recommendation as a link prediction problem taking into consideration a patient's diagnosis 


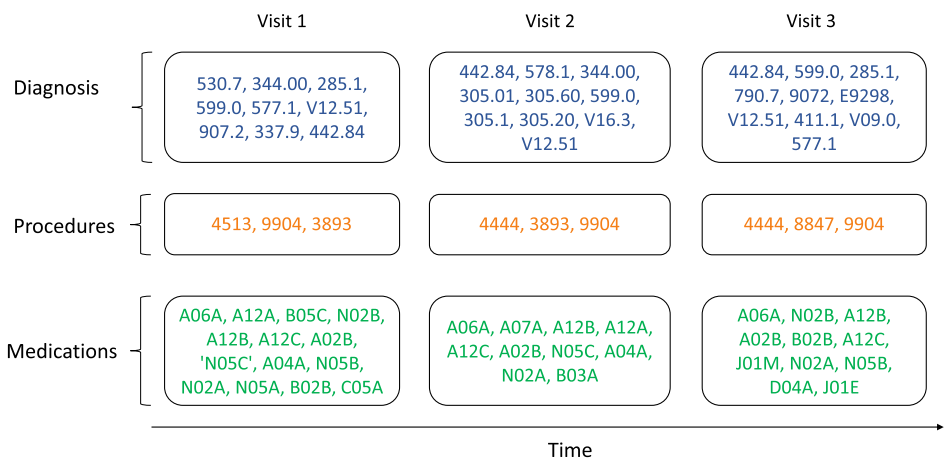

Fig. 1. Sample EHR depicting a sequence of three visits.

and adverse drug reactions. Wang et al. [43] also exploit demographics, diagnosis, and medication information and fuse them with a trilinear method. All these approaches ignore patients' diagnosis and so forth from the past visits, leading to loss in accuracy and hence do not personalize well.

Longitudinal recommendation. This class of recommender systems aims to exploit the temporal dependencies among a patient's past visits. Sun et al. [37] cluster the medical records of patients' past visits into cohorts based on their treatment similarities to find patterns in the treatment regimens.

Bajor and Lasko [2] propose to use RNNs to predict medications from a patient's clinical history and billing codes in the EHR. Although their model can predict if a drug is being used by a patient, it is unable to recommend multiple medications for complex disease conditions. Choi et al. [5] propose an attention-based predictive model to perform various tasks such as disease and medication prediction.

Le et al. [21] introduce DMNC, which infuses the concept of memory augmented neural networks [36] with RNNs to help in the handling of long-range dependencies by incorporating two memory modules for medication recommendation. All these works ignore drug interactions.

Shang et al. [30] propose a system called GameNet, which considers patients' longitudinal visit history and drug interactions. They use dynamic memory and GCNs to personalize the medication recommendations. CompNet [45] is another GCN-based medication recommender framework that combines reinforcement learning with a relational GCN. However, GCN remains a black-box, although attempts have been made to provide explanations via feature visualization [46]. Therefore, these medication recommender systems are unable to provide justification for the recommendations.

\section{METHODOLOGY}

The EHR of a patient can be represented as a sequence of $T$ visits. Figure 1 shows a sample EHR of a patient with three visits. The $i^{t h}$ visit is given by

$$
\boldsymbol{x}_{i}=\left[\boldsymbol{d}_{i}, \boldsymbol{p}_{i}, \boldsymbol{m}_{i}\right],
$$

where $\boldsymbol{d}_{i}, \boldsymbol{p}_{i}, \boldsymbol{m}_{i}$ are multi-hot vectors capturing the diagnosis, procedure, and medication, respectively, $i=1,2, \ldots, T$. Diagnosis and procedures are encoded with the ICD-9 system [34], whereas medications are encoded with the ATC classification [1]. The total number of diagnosis, procedure, and medications are denoted as $N_{d}, N_{p}$, and $N_{m}$, respectively.

We represent the drug co-occurrences in EHRs and drug interactions in the DDI database [40] as two graphs $A_{C}$ and $A_{D}$, respectively. Nodes in these graphs represent drugs, and an edge between 


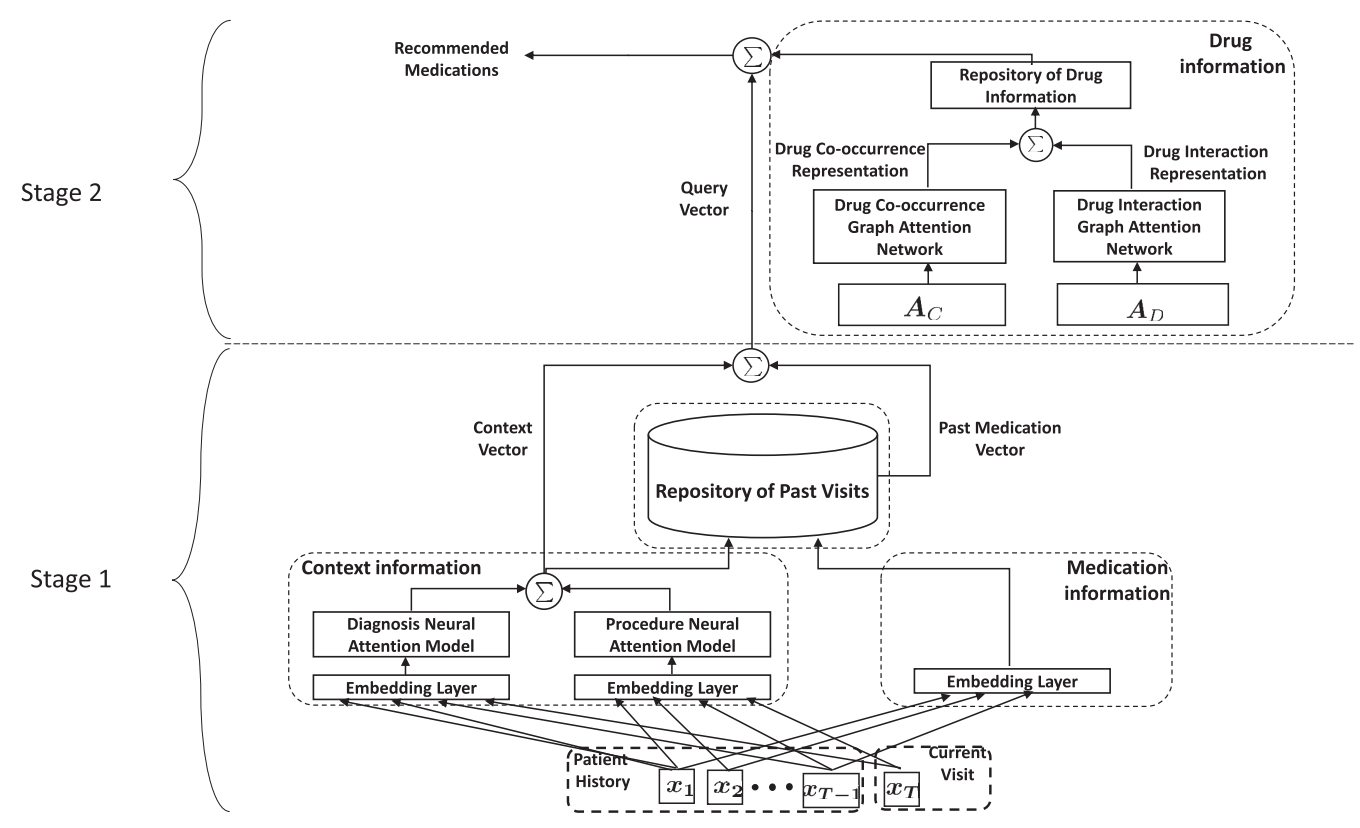

Fig. 2. Overview of the proposed recommender system PREMIER.

them defines their relationship. An edge in the drug co-occurrence graph indicates that two drugs have been prescribed together in the EHR, whereas an edge in the drug interaction graph implies that two drugs form a reactive pair.

Given a patient with past visits $\left[\boldsymbol{x}_{1}, \boldsymbol{x}_{2}, \ldots, \boldsymbol{x}_{T-1}\right]$, the diagnosis $d_{T}$ and procedure $p_{T}$ codes for the current visit, together with the drug co-occurrence $\boldsymbol{A}_{C}$ and drug interaction information $\boldsymbol{A}_{D}$, the task is to predict and justify a list of personalized medications for the patient's current visit $\boldsymbol{x}_{T}$.

We observe that a clinician typically refers to the diagnosis, procedures, drug-drug interaction, and prescriptions of a patient's past visits (along with diagnosis and procedures of the current visit) when deciding on the appropriate medications for the current visit. Further, this information may have different weights depending on their relevance to the current visit's diagnosis and procedures. This motivated us to develop a two-stage recommender system called PREMIER as shown in Figure 2. The first stage is designed to handle the relationship between the patient's historical visit record information and the current visit record, whereas the second stage leverages GATs to capture the complex information between drugs.

Two neural attention models are used in the first stage: one for procedures and another for diagnosis. This configuration allows us to alleviate the problem of missing modalities, which are common in the EHR. For example, we have patients whose diagnosis codes are available but the procedure codes are absent because the procedures are not recorded or no procedure has been ordered for the patient. Each neural attention model has two levels to learn the importance of the various visits, and the importance of the diagnosis (or procedures) of these visits to the current visit. This allows varying attention at the visit level, as well as at the diagnosis or procedure level within a visit. These attention weights provide some degree of explanation as opposed to standard RNNs, which are hard to interpret. The output of the neural attention models are concatenated to form a context vector. This context vector, together with the prescribed medications, are also stored in a key-value format inside a repository of past visits. This approach helps us retrieve the information on prescribed medications of the past visits of a patient based on the similarity between the 
Table 1. Notations Used in PREMIER

\begin{tabular}{|l|l|}
\hline Notation & \multicolumn{1}{|c|}{ Description } \\
\hline$N_{d}$ & Total number of diagnosis \\
$N_{p}$ & Total number of procedures \\
$N_{m}$ & Total number of medications \\
\hline $\boldsymbol{d}_{i} \in \mathbb{R}^{N_{d}}$ & Multi-hot vector of diagnosis codes \\
$\boldsymbol{p}_{i} \in \mathbb{R}^{N_{p}}$ & Multi-hot vector of procedure codes \\
$\boldsymbol{m}_{i} \in \mathbb{R}^{N_{m}}$ & Multi-hot vector of medication codes \\
\hline $\boldsymbol{A}_{C} \in \mathbb{R}^{N_{m} \times N_{m}}$ & Adjacency matrix of drug co-occurrence \\
$\boldsymbol{A}_{D} \in \mathbb{R}^{N_{m} \times N_{m}}$ & Adjacency matrix of drug interaction \\
\hline$Z_{C} \in \mathbb{R}^{64 \times N_{m}}$ & Drug co-occurrence representation \\
$Z_{D} \in \mathbb{R}^{64 \times N_{m}}$ & Drug interaction representation \\
\hline $\boldsymbol{\alpha} \in \mathbb{R}^{T}$ & Visit-level importance vector \\
$\boldsymbol{\beta} \in \mathbb{R}^{64 \times T}$ & Variable-level importance vector \\
\hline $\boldsymbol{r}^{d} \in \mathbb{R}^{64}$ & Response vector for diagnosis \\
$\boldsymbol{r}_{\in} \mathbb{R}^{64}$ & Response vector for procedures \\
$\boldsymbol{c} \in \mathbb{R}^{64}$ & Context vector \\
$\boldsymbol{v} \in \mathbb{R}^{64}$ & Visit history vector \\
$\boldsymbol{q} \in \mathbb{R}^{64}$ & Query vector \\
$\boldsymbol{o} \in \mathbb{R}^{64}$ & Output vector \\
\hline$\hat{\boldsymbol{y}} \in \mathbb{R}^{N_{m}}$ & Medication recommendation \\
\hline
\end{tabular}

current context vector and the context vector of the past visits. This helps in personalizing the recommended medications. Next, we extract a past medication vector from this repository and combine it with the context vector to generate a query vector that incorporates information from the present and past diagnosis, as well as procedures along with the past prescribed medications. This query vector depicts the patient representation that is used to retrieve drug interaction and co-occurrence information from the second stage.

Graph attention networks [42] are employed in the second stage to learn the embeddings for the drug co-occurrence and drug interaction information. This allows us to incorporate the frequency of drug co-occurrences and the severity of drug interactions, which improves the accuracy of the predicted medications while reducing adverse drug interactions, as well as providing justification for the recommendations. The output of the GATs are stored in the repository of drug information. The query vector generated in the first stage is used to retrieve the relevant drug information to personalize the medications recommended.

We describe the details of PREMIER in the following sections. Table 1 summarizes the notations used.

\subsection{Stage 1: Create Query Vector}

Here we describe the details of the steps in stage 1 of PREMIER. Given a patient with $T$ visits, we linearly embed the diagnosis, procedure, and past medication vectors into a 64-dimensional space to learn the distributed representations, denoted as $\boldsymbol{d}_{i}^{e}, \boldsymbol{p}_{i}^{e}, \boldsymbol{m}_{i}^{e}$, for $1 \leq i \leq T$ by using the embedding matrices $E^{d}, E^{p}, E^{m}$, respectively. These embedding matrices represent the embedding layers and are learned during the training phase. The embeddings of the diagnosis and procedure vectors are passed to the respective neural attention models as shown in Figure 3.

Consider a neural attention model similar to that in the work of Choi et al. [5] that takes as input the diagnosis embedding. We model the visit-level importance for diagnosis as $\boldsymbol{\alpha}^{\boldsymbol{d}}$ where 


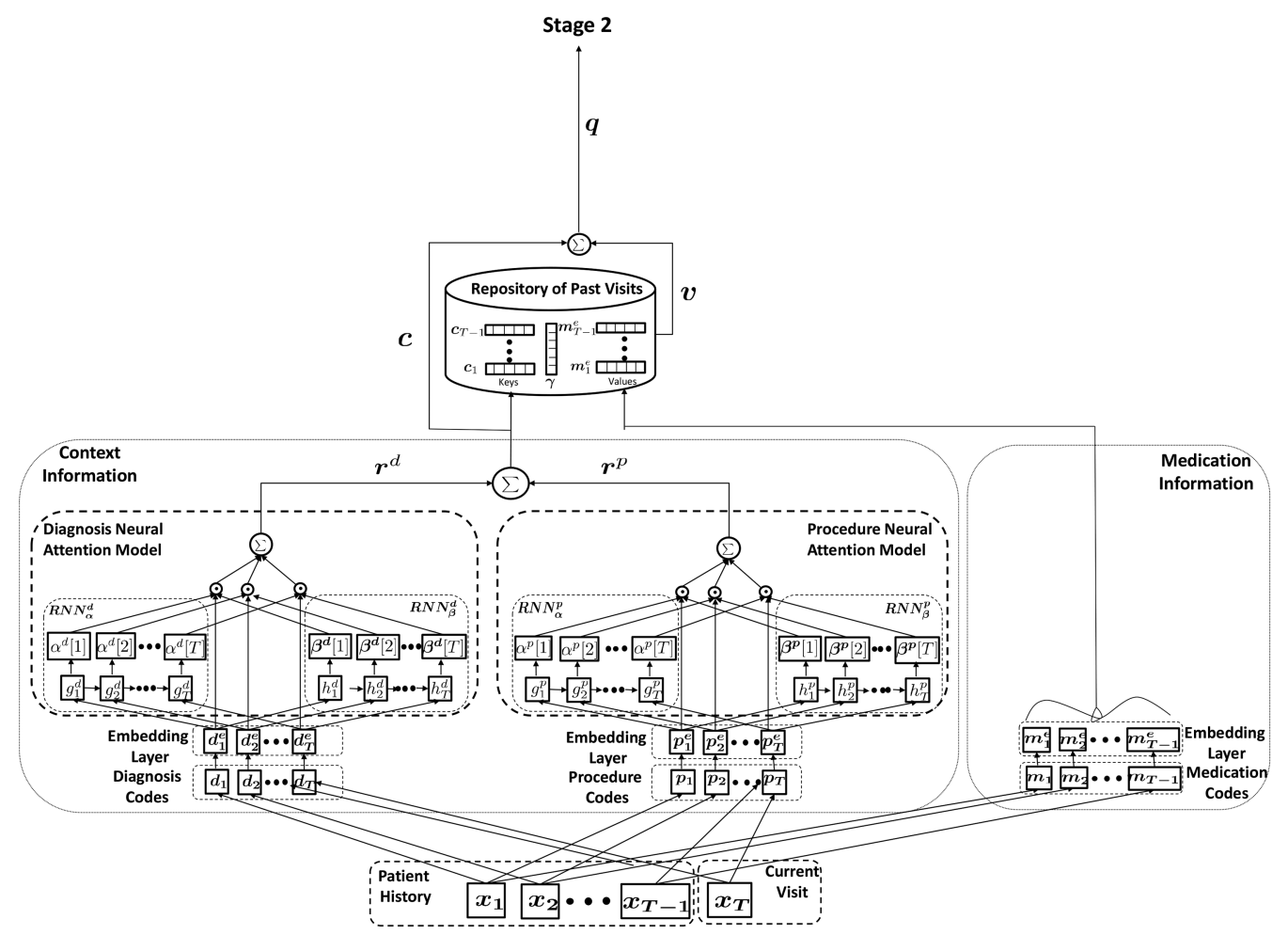

Fig. 3. Details of the first stage of PREMIER.

the $i^{t h}$ entry, $\alpha^{d}[i]$, is a scalar value depicting the weight for the $i^{t h}$ visit, $1 \leq i \leq T$. Separately, we also model the diagnosis-level importance of a visit as $\boldsymbol{\beta}^{\boldsymbol{d}}$ where the $j^{\text {th }}$ entry, $\boldsymbol{\beta}^{\boldsymbol{d}}[j]$, is a vector depicting the weights of the various diagnosis code embeddings, $1 \leq j \leq T$. We utilize $R N N_{\alpha}^{d}$ to compute the vector $\boldsymbol{\alpha}^{\boldsymbol{d}}$ as follows:

$$
\begin{aligned}
& \boldsymbol{g}_{1}, \boldsymbol{g}_{2}, \ldots, \boldsymbol{g}_{T}=R N N_{\boldsymbol{\alpha}}^{d}\left(\boldsymbol{d}_{1}^{e}, \boldsymbol{d}_{2}^{e}, \ldots, \boldsymbol{d}_{T}^{e}\right) \\
& \boldsymbol{\alpha}^{\boldsymbol{d}}=\operatorname{softmax}\left(\phi\left(\boldsymbol{g}_{1}\right), \phi\left(\boldsymbol{g}_{2}\right), \ldots, \phi\left(\boldsymbol{g}_{T}\right)\right),
\end{aligned}
$$

where each $g_{i}$ is a hidden layer in $R N N_{\alpha}^{d}$ and $\phi$ is a linear transformation function in which the weight vector $\in \mathbb{R}^{64 \times 1}$. Here, we use softmax to obtain the level of contribution of the visits, with the values ranging between 0 and 1 .

Similarly, we use a second $R N N_{\beta}^{d}$ to obtain the vector $\boldsymbol{\beta}^{d}[j]$ :

$$
\begin{gathered}
\boldsymbol{h}_{1}, \boldsymbol{h}_{2}, \ldots, \boldsymbol{h}_{T}=R N N_{\boldsymbol{\beta}}^{d}\left(\boldsymbol{d}_{1}^{e}, \boldsymbol{d}_{2}^{e}, \ldots, \boldsymbol{d}_{T}^{e}\right) \\
\boldsymbol{\beta}^{\boldsymbol{d}}[j]=\tanh \left(\psi\left(\boldsymbol{h}_{j}\right)\right), \quad j=1,2, \ldots, T,
\end{gathered}
$$

where each $\boldsymbol{h}_{j}$ is a hidden layer in $R N N_{\beta}^{d}$ and $\psi$ is a linear transformation function in which the weight matrix $\in \mathbb{R}^{64 \times 64}$. We use tanh in Equation (2) such that the value lies between -1 and 1 to capture both the negative and positive contribution of a diagnosis. A diagnosis has a negative contribution when it excludes the use of some medication for another diagnosis. 
We determine the response vector $\boldsymbol{r}^{d} \in \mathbb{R}^{64 \times 1}$ of diagnosis for the current visit $T$ as follows:

$$
\boldsymbol{r}^{d}=\sum_{i=1}^{T} \alpha^{d}[i] \boldsymbol{\beta}^{\boldsymbol{d}}[i] \odot \boldsymbol{d}_{i}^{e},
$$

where $\odot$ denotes element-wise multiplication.

The response vector for the procedure of the current visit $\boldsymbol{r}^{p}$ can be obtained similarly. With this, we can define the current visit context vector $c$ as follows:

$$
\boldsymbol{c}=\boldsymbol{r}^{d}+w_{1} \boldsymbol{r}^{p}
$$

where $w_{1} \in \mathbb{R}$ is a parameter that depicts the importance of procedure information in the context vector.

We insert $\boldsymbol{c}$ into a repository of the context vectors for a patient's past visits and the corresponding embedded medications that are stored in the form of key-value pairs where the key is the context vector and the value is the embedded medications.

Given the context vector $c$ for the current visit $T$, we compute the attention on the context vectors of the previous visits $\boldsymbol{c}_{i}, i \in[1, T-1]$ stored in the repository as follows:

$$
\boldsymbol{\gamma}=\operatorname{softmax}\left(\boldsymbol{c}_{1} \cdot \boldsymbol{c}, \boldsymbol{c}_{2} \cdot \boldsymbol{c}, \ldots, \boldsymbol{c}_{T-1} \cdot \boldsymbol{c}\right) .
$$

With this attention $\boldsymbol{\gamma} \in \mathbb{R}^{T-1}$, we obtain the past medication vector $\boldsymbol{v}$ as follows:

$$
v=\gamma \cdot \boldsymbol{m}^{e} \text {. }
$$

Finally, we combine the context vector and the past medication vector to form the query vector $\boldsymbol{q} \in \mathbb{R}^{64}$ as follows:

$$
\boldsymbol{q}=\boldsymbol{c}+w_{2} \boldsymbol{v},
$$

where $w_{2} \in \mathbb{R}$ is a parameter that depicts the importance of the past medications in the query vector.

\subsection{Stage 2: Retrieve Drug Information}

We use the query vector obtained in stage 1 to retrieve the drug co-occurrence and drug interaction information as shown in Figure 4.

We use two graph attention networks-one for drug co-occurrences and another for drug interactions-to learn an efficient representation for recommendation (see Figure 4). Each graph attention network has two layers. The first layer utilizes two attention heads, whereas the second layer has one attention head.

The input to the first GAT is the drug co-occurrence graph $A_{C}$. Given a drug $j$, let $S_{j}$ be the set of nodes in $A_{C}$ depicting the drugs that co-occur with $j$. The output representation of the $j^{t h}$ unit in the first layer of GAT is given by

$$
\boldsymbol{u}_{j}^{1}=\|_{b=1}^{2} \sigma\left(\sum_{n_{k} \in S_{j}} \delta_{j k}^{b} \boldsymbol{W}^{b} n_{k}\right)
$$

where $\|$ denotes concatenation, $\boldsymbol{W}^{b} \in \mathbb{R}^{64 \times N_{m}}$ is the embedding matrix, and $\delta_{j k}^{b}$ is the attention weight between the pair of drugs $j$ and $k$ for the $b^{\text {th }}$ attention head computed as follows:

$$
\delta_{j k}^{b}=\frac{\exp \left(\operatorname{LeakyReLU}\left(\boldsymbol{a}^{\prime}\left[\boldsymbol{W}^{b} n_{j} \| \boldsymbol{W}^{b} n_{k}\right]\right)\right)}{\sum_{n \in S_{j}} \exp \left(\operatorname{LeakyReLU}\left(\boldsymbol{a}^{\prime}\left[\boldsymbol{W}^{b} n_{j} \| \boldsymbol{W}^{b} n\right]\right)\right)},
$$

where $a^{\prime}$ is the transpose of the weight vector of a single-layer feed-forward neural network. 


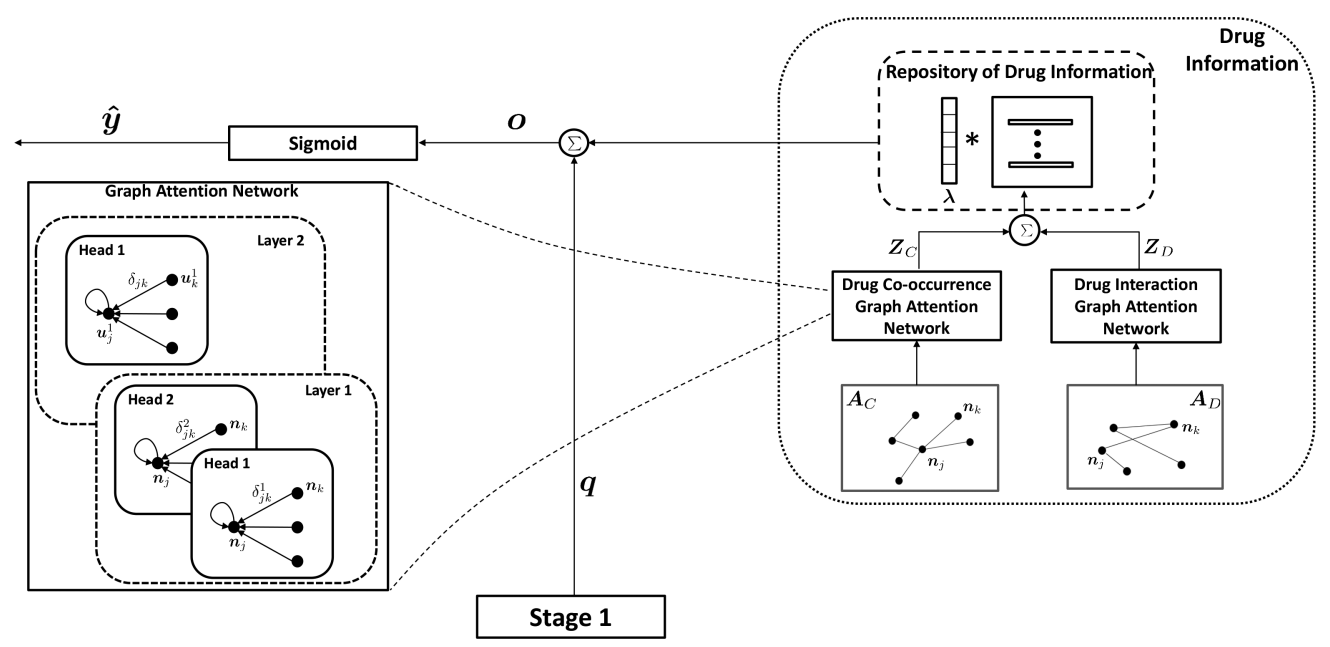

Fig. 4. Details of the second stage of PREMIER.

The final representation of a drug $j$ is obtained from the second layer of the graph attention network as follows:

$$
\boldsymbol{u}_{j}^{2}=\sigma\left(\sum_{k \in \mathcal{S}_{j}} \delta_{j k} \boldsymbol{W} \boldsymbol{u}_{k}^{1}\right),
$$

where $W \in \mathbb{R}^{64 \times 128}$ is the embedding matrix.

With this, we can generate the drug co-occurrence representation $Z_{C} \in \mathbb{R}^{64 \times N_{m}}$. In the same manner, the drug interaction graph attention network is used to obtain the drug interaction representation $Z_{D} \in \mathbb{R}^{64 \times N_{m}}$.

We can compute the attention $\lambda$ based on these representations and the query vector $q$ as follows:

$$
\lambda=\operatorname{softmax}\left(\left(Z_{C}+w_{3} Z_{D}\right)^{\prime} \cdot \boldsymbol{q}\right),
$$

where $\left(Z_{C}+w_{3} Z_{D}\right)^{\prime}$ is the transpose of $\left(Z_{C}+w_{3} Z_{D}\right)$ and $w_{3} \in \mathbb{R}$ is a parameter that depicts the importance of the drug interaction.

The final output vector $\boldsymbol{o}$ is obtained as

$$
\boldsymbol{o}=\boldsymbol{q}+w_{4}\left(\left(Z_{C}+w_{3} Z_{D}\right) \cdot \lambda\right)
$$

where $w_{4} \in \mathbb{R}$ is a parameter depicting the importance of drug information.

We obtain a vector $\hat{\boldsymbol{y}}$ for the current visit $T$ by passing $\boldsymbol{o}$ through a linear transformation followed by a sigmoid function. A medication $k$ is considered predicted if $\hat{\boldsymbol{y}}[k]>0.5, k \in\left\{1, N_{m}\right\}$. Using Equations (4) and (7), we have

$$
\hat{\boldsymbol{y}}=F\left(\boldsymbol{r}^{d}+w_{1} \boldsymbol{r}^{p}+w_{2} \boldsymbol{v}+w_{4}\left(\left(Z_{C}+w_{3} Z_{D}\right) \cdot \boldsymbol{\lambda}\right)\right) .
$$

\subsection{Justify Recommended Medications}

In this section, we describe how we justify the recommended medications by determining the percentage of contribution from diagnosis, procedures, and previously prescribed medications, and highlighting the top contributors. 
Let us consider the $j^{\text {th }}$ medication recommended at the current visit $T$. Combining the first term in Equation (13) with Equation (3), we obtain the contribution due to a patient's diagnosis:

$$
\text { score_diagnosis }=\|_{i=1}^{T}\left(\alpha^{d}[i] E^{F}[j,:]\left(\boldsymbol{\beta}^{d}[i] \odot \boldsymbol{E}^{d}[:, k]\right) d_{i}[k]\right),
$$

where $d_{i}[k]$ denotes a specific diagnosis in the $i^{t h}$ visit where $k \in\left\{1, N_{d}\right\}, E^{d}$ denotes the embedding matrix for $\boldsymbol{d}_{i}$, and $\boldsymbol{E}^{F}$ is the weight matrix used in the linear transformation function $F$.

Similarly, we can compute the contribution due to the procedures undergone by the patient from the second term in Equation (13) and Equation (3) as follows:

$$
\text { score_procedure }=\|_{i=1}^{T}\left(w_{1} \alpha^{p}[i] E^{F}[j,:]\left(\boldsymbol{\beta}^{p}[i] \odot \boldsymbol{E}^{p}[:, k]\right) p_{i}[k]\right),
$$

where $p_{i}[k]$ denotes a specific procedure in the $i^{t h}$ visit where $k \in\left\{1, N_{p}\right\}$ and $E^{p}$ denotes the embedding matrix for $\boldsymbol{p}_{i}$.

To determine the contribution due to the past prescribed medications, we substitute Equation (6) to the third term in Equation (13) as follows:

$$
\text { score_medication }=\|_{i=1}^{T-1}\left(w_{2} \gamma_{t}[i]\left(\boldsymbol{E}^{F}[j,:] \cdot \boldsymbol{E}^{m}[:, k]\right) m_{i}[k]\right)
$$

where $m_{i}[k]$ denotes the specific medication prescribed in the $i^{t h}$ visit where $k \in\left\{1, N_{m}\right\}$ and $\boldsymbol{E}^{m}$ denotes the embedding matrix for $\boldsymbol{m}_{i}$.

We calculate the contributions due to drug co-occurrences and drug interactions as follows:

$$
\begin{gathered}
\text { score_occurrence }=w_{4} E^{F}[j,:] Z_{C} \cdot \lambda \\
\text { score_interaction }=w_{4} w_{3} E^{F}[j,:] Z_{D} \cdot \lambda,
\end{gathered}
$$

where $Z_{C}$ and $Z_{D}$ are obtained from graph attention networks as described in Section 3.2.

The final contributions are obtained by normalizing the five scores such that they add up to 1 . We rank these scores and use the top two scores as justification for the recommendations.

\subsection{Loss Functions}

There are three loss functions in PREMIER: binary cross entropy loss, multi-label hinge loss, and drug-drug interaction loss. Since this is a sequential multi-label prediction task, we use binary cross entropy loss $\mathrm{L}_{\text {entropy }}$ defined as:

$$
\mathrm{L}_{\text {entropy }}=-\sum_{i=1}^{T} \sum_{k=1}^{N_{m}}\left(\boldsymbol{y}_{i}[k] \log \hat{\boldsymbol{y}}_{i}[k]+\left(1-\boldsymbol{y}_{i}[k]\right) \log \left(1-\hat{\boldsymbol{y}}_{i}[k]\right)\right),
$$

where $N_{m}$ is the total number of medications, and $\hat{\boldsymbol{y}}_{i}[k]$ and $\boldsymbol{y}_{i}[k]$ are the $k^{\text {th }}$ predicted and ground truth medication for the $i^{t h}$ visit, respectively.

Let $U$ be the set of positions where the entry in the ground truth medication vector is 1 . We define a multi-label hinge loss to ensure that the score of the correctly predicted class is higher than the scores of the other predicted class as follows:

$$
\mathrm{L}_{\text {hinge }}=\sum_{i=1}^{T} \sum_{k=1}^{N_{m}} \sum_{u \in U} \frac{\max \left(0,1-\left(\hat{\boldsymbol{y}}_{i}[u]-\hat{\boldsymbol{y}}_{i}[k]\right)\right)}{|U|},
$$

This loss helps to increase the margin between the correctly and wrongly predicted classes, which in turn facilitates the use of a threshold of 0.5 to decide whether $\hat{\boldsymbol{y}}_{i}[k]$ is considered predicted (recall Section 3.2). 
The drug-to-drug interaction loss, denoted as $\mathrm{L}_{\text {adverse, }}$, penalizes the pairs of drugs that have adverse interactions to ensure safe recommendation and is defined as

$$
\mathrm{L}_{\text {adverse }}=\sum_{i=1}^{T} \sum_{j=1}^{N_{m}} \sum_{k=1}^{j-1}\left(\boldsymbol{A}_{D} \odot \hat{\boldsymbol{y}}_{i}^{\prime} \hat{\boldsymbol{y}}_{i}\right)[j, k] .
$$

For example, analgesics (N02B) like paracetamol and folic acid (B03B) may cause bronchitis and lung edema and should not be prescribed together. Hence, the loss in Equation (16) will be high for this pair of drugs, and the system will learn not to recommend this pair.

To ensure that the recommendation is accurate and safe at the same time, the final objective function is a weighted sum of the three losses:

$$
\mathrm{L}_{\text {combine }}=\gamma_{1} * \mathrm{~L}_{\text {entropy }}+\gamma_{2} * \mathrm{~L}_{\text {hinge }}+\left(1-\gamma_{1}-\gamma_{2}\right) * \mathrm{~L}_{\text {adverse }} \text {. }
$$

\section{PERFORMANCE STUDY}

In this section, we describe the experiments we carry out to evaluate the performance of PREMIER for medication recommendations. The implementation is done in PyTorch, and the models are trained on two NVIDIA Titan RTX GPUs.

The evaluation metrics for the accuracy of the predictions are the AUC [30], F1 [30], and Jaccard similarity coefficient [47]. We also use the DDI metric proposed by Shang et al. [30] to measure the percentage of pairwise drug interactions in the list of predicted medications. A low value for DDI is desirable since it indicates fewer drug interactions. Since our objective is to maximize the number of correctly predicted medications and minimize the drug interactions, we introduce a metric called DScore that captures the tradeoff between accuracy and adverse reactions:

$$
\text { DScore }=\frac{2 \times \mathrm{ACC} \times(1-\mathrm{DDI})}{\mathrm{ACC}+(1-\mathrm{DDI})},
$$

where ACC denotes an accuracy measure.

Depending on whether we use AUC, F1, or Jaccard as the accuracy measure, we have three variants of DScore, denoted as DScore $\mathrm{AUC}_{\mathrm{C}}, \mathrm{DScore}_{\mathrm{F} 1}$, and $\mathrm{DScore}_{\mathrm{Jac}}$, respectively. A high DScore indicates that the set of predicted medications is close to the set of prescribed medications with a low drug-to-drug interaction rate.

\subsection{Experiment Datasets}

We use the following datasets in our experiments.

MIMIC-III [16]. MIMIC-III is the largest publicly available EHR dataset, which contains clinical data for 7,870 neonates (infants) and 38,597 adults admitted to the ICU between 2001 and 2008, and captures attributes such as lab reports, vital signs, and medications. This dataset uses the ICD-9 coding system for diagnosis, and the NDC (National Drug Code) system for medications.

PRIVATE. This is a 1-year outpatient proprietary dataset collected between 2014 and 2015. Compared to the inpatient MIMIC III, this dataset has a larger patient cohort, but the number of diagnoses per patient is much fewer and there are no procedure codes.

We pre-process the dataset to filter out those patients who have only one visit. We create the drug interaction adjacency matrix $A_{D}$ from the TWOSIDES dataset [40] using the top 40 interactions for each drug because these cover the major severe effects, similar to the work of Shang et al. [30]. Since the TWOSIDES dataset uses the ATC Third Level drug classification system, we transform the medication names to ATC coding and create the drug co-occurrence adjacency matrix $A_{C}$. Table 2 gives the characteristics of the two datasets obtained. 
Table 2. Characteristics of Datasets

\begin{tabular}{|l|c|c|}
\hline Attribute & MIMIC-III & PRIVATE \\
\hline Number of patients & 6,350 & 13,640 \\
\hline Number of diagnoses & 1,958 & 11 \\
\hline Number of procedures & 1,430 & - \\
\hline Number of medications & 151 & 134 \\
\hline Average number of visits per patient & 2.36 & 3.42 \\
\hline Average number of diagnoses per visit & 13.63 & 2.37 \\
\hline Average number of procedures per visit & 4.53 & - \\
\hline Average number of medications per visit & 19.85 & 5.04 \\
\hline Maximum number of diagnoses per visit & 39 & 7 \\
\hline Maximum number of procedures per visit & 32 & - \\
\hline Maximum number of medications per visit & 55 & 8 \\
\hline
\end{tabular}



(a) Dimension of embedding layer

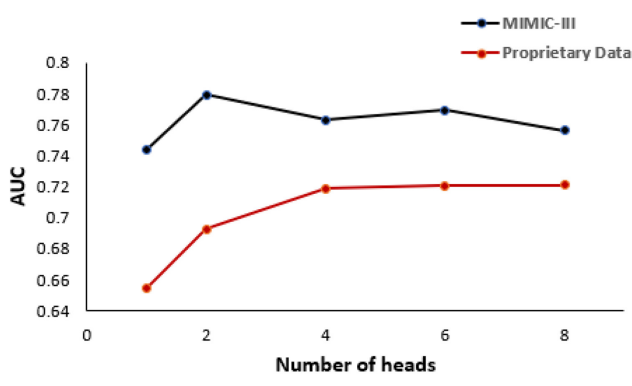

(b) Attention head of the graph attention network

Fig. 5. Effect of embedding dimension and attention heads on PREMIER.

We divide the dataset into training, validation, and test sets in the ratio of 4:1:1. The embedding size for our model is fixed at 64, and training is done using Adam [18]. The size of the hidden layers for the neural attention models is set to 64. Gated recurrent units (GRUs) are used for RNN blocks, since it is efficient and widely used for modeling sequential dependencies.

For MIMIC-III, we apply a dropout of 0.4 on the input embedding layer. The graph attention network consists of two layers with two attention heads in the first layer and one attention head in the second layer. The parameter values are set to be $\gamma_{1}=0.79, \gamma_{2}=0.01, w_{1}=1, w_{2}=1, w_{3}=0.3$, and $w_{4}=1$ based on the validation set. The learning rate is 0.0001 , and the best-performing model is chosen based on the performance on the validation set after 40 epochs.

For PRIVATE, the dropout is 0.6 and the graph attention network consists of two layers with four attention heads in the first layer and one attention head in the second layer. The parameter values are $\gamma_{1}=0.75, \gamma_{2}=0.05, w_{1}=1, w_{2}=1, w_{3}=0.225$, and $w_{4}=1$. The learning rate of 0.0002 and the best-performing model is chosen on the validation set after 30 epochs.

All the reported results are for the test sets.

\subsection{Sensitivity Experiments}

We first examine the effect of the parameters on the performance of PREMIER. We vary the embedding dimensions and report the performance in terms of AUC. Figure 5(a) shows that the best model performance was achieved when the dimension is 64 for both MIMIC-III and 



(a) MIMIC-III
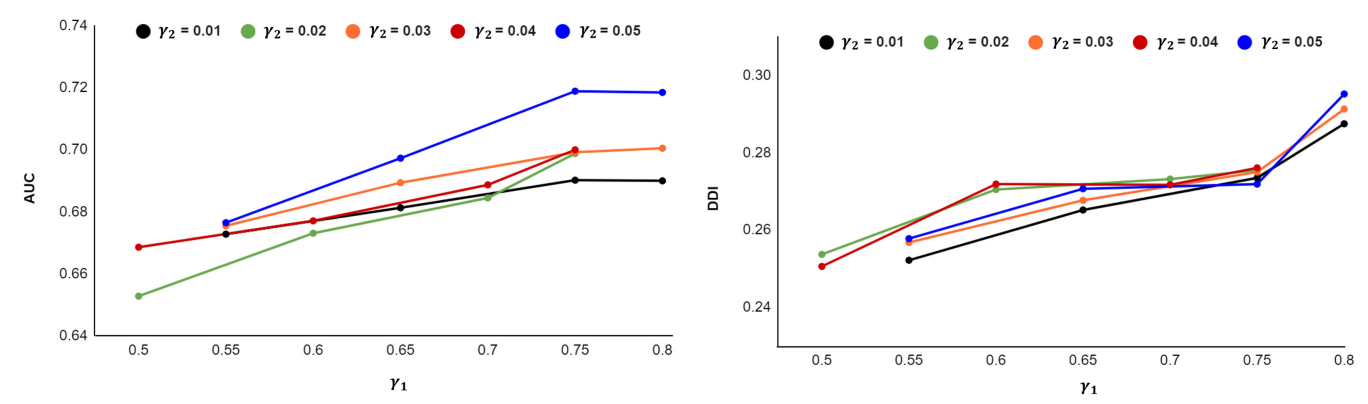

(b) PRIVATE

Fig. 6. Effect of loss weights on PREMIER.

PRIVATE. Figure 5(b) shows the performance of the system when we vary the attention heads in the first layer of the graph attention networks. Two attention heads gives the best performance for MIMIC-III, whereas the best performance on PRIVATE is achieved by four attention heads. Therefore, we fix the embedding dimension to 64 for both the datasets, whereas the number of heads is set to 2 and 4 for MIMIC-III and PRIVATE, respectively, for the rest of the experiments.

Figure 6(a) shows the AUC and DDI of PREMIER on the MIMIC-III dataset when we vary the loss weights $\gamma_{1}$ and $\gamma_{2}$. We observe that as $\gamma_{1}$ increases, the AUC is higher while the DDI degrades. The best performance with least tradeoff is obtained for the combination $\gamma_{1}=0.79$ and $\gamma_{2}=0.01$. Figure 6(b) shows similar analysis on the PRIVATE dataset with the best tradeoff achieved when $\gamma_{1}=0.75$ and $\gamma_{2}=0.05$.

\subsection{Comparative Experiments}

We compare PREMIER with several baseline approaches as well as state-of-art medication recommender systems:

- K-frequent: This is a rule-based approach that uses the most frequently occurring medications in the EHR prescriptions for the various diagnoses.

- LR [22]: Here, we use the binary relevance implementation of logistic regression with L2 regularization since our recommendation is multi-label in nature.

- LEAP [47]: This is a sequential decision-making system that provides recommendations based on the current visit.

- DMNC [21]: This is a dual memory neural computer that prescribes a set of medications based on the information of the past visits stored in the external memory components. 
Table 3. Results of the Comparative Study

(a) MIMIC-III

\begin{tabular}{|c|c|c|c|c|c|c|c|c|c|}
\hline \multirow{2}{*}{ Methods } & \multicolumn{4}{|c|}{ Accuracy } & $\begin{array}{c}\text { Drug Int- } \\
\text { eraction }\end{array}$ & \multicolumn{3}{c|}{ Tradeoff } \\
\cline { 2 - 11 } & AUC & Recall & Precision & F1 & Jaccard & DDI & DScore AUC & DScore \\
F1 & DScore Jac \\
\hline \hline LR & 0.655 & 0.612 & 0.617 & 0.614 & 0.455 & 0.079 & 0.765 & 0.737 & 0.609 \\
\hline K-frequent & 0.702 & 0.617 & 0.616 & 0.617 & 0.464 & 0.079 & 0.797 & 0.739 & 0.617 \\
\hline LEAP & 0.642 & 0.606 & 0.617 & 0.606 & 0.444 & $\mathbf{0 . 0 6 7}$ & 0.761 & 0.735 & 0.601 \\
\hline DMNC & 0.664 & 0.637 & 0.601 & 0.619 & 0.445 & 0.090 & 0.768 & 0.737 & 0.598 \\
\hline GameNet & 0.747 & 0.727 & 0.598 & 0.656 & 0.500 & 0.079 & 0.825 & 0.766 & 0.648 \\
\hline CompNet & 0.754 & 0.727 & 0.603 & 0.659 & 0.505 & 0.078 & 0.830 & 0.779 & 0.658 \\
\hline PREMIER & $\mathbf{0 . 7 8 0}^{*}$ & $\mathbf{0 . 7 5 3}^{*}$ & $\mathbf{0 . 6 2 2}^{*}$ & $\mathbf{0 . 6 8 1}^{*}$ & $\mathbf{0 . 5 2 7}^{*}$ & 0.075 & $\mathbf{0 . 8 4 6}^{*}$ & $\mathbf{0 . 7 8 5}^{*}$ & $\mathbf{0 . 6 7 1}^{*}$ \\
\hline
\end{tabular}

(b) PRIVATE

\begin{tabular}{|c|c|c|c|c|c|c|c|c|c|}
\hline \multirow{2}{*}{ Methods } & \multicolumn{4}{|c|}{ Accuracy } & $\begin{array}{c}\text { Drug Int- } \\
\text { eraction }\end{array}$ & \multicolumn{3}{|c|}{ Tradeoff } \\
\cline { 2 - 11 } & AUC & Recall & Precision & F1 & Jaccard & DDI & DScore AUC & DScore \\
& D1 & DScore Jac \\
\hline \hline LR & 0.565 & 0.401 & 0.501 & 0.446 & 0.329 & 0.346 & 0.606 & 0.530 & 0.438 \\
\hline K-frequent & 0.616 & 0.542 & 0.594 & 0.566 & 0.439 & 0.321 & 0.646 & 0.618 & 0.533 \\
\hline LEAP & 0.588 & 0.454 & 0.603 & 0.518 & 0.390 & $\mathbf{0 . 2 6 0}$ & 0.655 & 0.609 & 0.511 \\
\hline GameNet & 0.693 & 0.620 & 0.599 & 0.609 & 0.485 & 0.302 & 0.696 & 0.651 & 0.572 \\
\hline CompNet & 0.697 & 0.626 & 0.605 & 0.616 & 0.499 & 0.288 & 0.705 & 0.660 & 0.587 \\
\hline PREMIER & $\mathbf{0 . 7 1 9}^{*}$ & $\mathbf{0 . 6 5 0}^{*}$ & $\mathbf{0 . 6 3 2}^{*}$ & $\mathbf{0 . 6 4 1}^{*}$ & $\mathbf{0 . 5 4 0}^{*}$ & 0.272 & $\mathbf{0 . 7 2 3}^{*}$ & $\mathbf{0 . 6 8 2}^{*}$ & $\mathbf{0 . 6 2 0}^{*}$ \\
\hline
\end{tabular}

The asterisk $\left(^{*}\right)$ indicates that the result is statistically significant when compared to the second best with a $p$-value $<$ 0.05 .

- GameNet [30]: This work models the patient data as a collection of his past visits together with drug interaction information.

- CompNet [45]: This work utilizes graph convolutional reinforcement learning to model the patients' visits along with drug interaction information.

Since the PRIVATE dataset does not have procedure codes, we adapt PREMIER, GameNet, and CompNet to run without procedure codes. We omit DMNC, as it cannot run without procedure codes.

Table 3(a) shows the results for the MIMIC-III test set with an inherent DDI of 0.0750 . We see that PREMIER achieves the best performance in terms of accuracy and DScores, as it takes into account the past prescribed medications and the varying importance of drug interactions. The improvement in recall can be attributed to our proposed patient representation that incorporates longitudinal visit history. Improvement in precision is due to PREMIER's ability to capture drug interaction severity through the use of GAT to model drug co-occurrence and drug interaction information. The one-way ANOVA [8] test shows that the improvements in AUC, Recall, Precision, F1, and Jaccard by PREMIER over CompNet are statistically significant with $p$-values of $9.6 \times 10^{-8}$, $1.3 \times 10^{-6}, 3.5 \times 10^{-5}, 2.7 \times 10^{-6}$, and $5.2 \times 10^{-5}$, respectively.

Although LEAP has the best DDI, it has the lowest DScores, indicating that its accuracy is the worst. Further, the $p$-value between PREMIER and LEAP is $0.063(>0.05)$, indicating that the improvement in DDI by LEAP is not significant. In fact, the DDI in the actual prescribed medications in the test set is 0.0750 , which is similar to that attained by PREMIER.

Table 3(b) gives the results for the PRIVATE dataset with an inherent DDI of 0.196. Again, we see that PREMIER remains the top performer in terms of accuracy and DScore, demonstrating the 
Table 4. Average Number of Recommended, Extra, and Missed Medications

\begin{tabular}{|c|c|c|c|c|c|c|}
\hline \multirow{2}{*}{ Methods } & \multicolumn{3}{|c|}{ MIMIC-III } & \multicolumn{3}{c|}{ PRIVATE } \\
\cline { 2 - 7 } & \# Average & \# Extra & \# Missed & \# Average & \# Extra & \# Missed \\
\hline LR & 14.21 & 3.11 & 9.50 & 1.87 & 0.41 & 1.29 \\
\hline K-frequent & 23.01 & 8.84 & 7.17 & 3.15 & 1.21 & 0.98 \\
\hline LEAP & 19.01 & 7.29 & 8.00 & 2.89 & 1.01 & 1.21 \\
\hline DMNC & 23.60 & 9.22 & 6.96 & - & - & - \\
\hline GameNet & 25.06 & 10.58 & 5.71 & 7.28 & 3.07 & 1.15 \\
\hline CompNet & 23.16 & 8.95 & 5.62 & 3.69 & 1.32 & 0.89 \\
\hline PREMIER & 22.65 & 7.55 & 5.49 & 4.27 & 1.02 & 0.53 \\
\hline
\end{tabular}

effectiveness of our approach. The improvements in AUC, Recall, Precision, F1, and Jaccard are statistically significant with $p$-values of $1.4 \times 10^{-6}, 1.6 \times 10^{-6}, 5.1 \times 10^{-6}, 8.2 \times 10^{-6}$, and $3.7 \times 10^{-4}$ , respectively. For DDI, we observe that the $p$-value between PREMIER and LEAP is $0.083(>0.05)$, indicating that the improvement in DDI by LEAP is not significant.

Number of recommended medications. Table 4 shows the average number of medications recommended, the average number of extra medications recommended, and the average number of medications that are missed per visit. In terms of the average number of medications recommended, we observe that LEAP and LR tend to recommend significantly fewer medications to patients, whereas GameNet often over-recommends in both datasets. In contrast, the average number of medications recommended by PREMIER is close to that in the datasets.

When we examine the extra medications recommended, we find the GameNet has the highest number of extra medications, indicating that not capturing the severity level of drug interaction may lead to over-recommendation. In contrast, the number of extra medications recommended by PREMIER is relatively low. This suggests that having different attention weights for various instances in multiple information sources while allowing the model to learn the varying severity of drug interactions helps boost the performance. For the missed medications, we see that LR misses the most number of medications, suggesting that considering information from previous visits is important. In contrast, PREMIER has the least number of missed medications per visit.

Effect of number of visits. We examine the impact of the number of visits on the performance of various methods. For this analysis, we use the cohort of patients in MIMIC-III with exactly five visits since there are very few patients with more than five visits. We exclude LEAP, LR, and Kfrequent from this analysis, as they do not use patients' visit information.

Figure 7 shows the results for MIMIC-III and PRIVATE, respectively. We observe that PREMIER outperforms other baselines in terms of AUC and DDI, indicating that PREMIER can effectively utilize patient visit history and strive to recommend safe medications without compromising on the accuracy aspect. PREMIER continues to be the best performer and remains robust even when there is few visit information, such as when the number of visits is less than 3.

Effect of visit-aware and diagnosis/procedure-aware attentions. We examine the effect of $\boldsymbol{\alpha}$ and $\boldsymbol{\beta}$ on the performance of PREMIER. For PREMIER $\boldsymbol{\beta}$, we ignore the visit-level attention by setting $\alpha[i]$ in Equation (3) to 1 . PREMIER $\boldsymbol{\alpha}$ is obtained by setting $\boldsymbol{\beta}[i]$ in Equation (3) to a vector whose entries are 1 . Table 5 shows the results for both datasets. We see that the proposed PREMIER that incorporates the attention weights from both visit-level and diagnosis/procedure-level yields the best performance. PREMIER $\boldsymbol{\beta}$ performs slightly better than PREMIER $\boldsymbol{\alpha}$, as $\boldsymbol{\beta}$ gives the diagnosis/procedure-level importance, which is more critical for medication recommendation. 

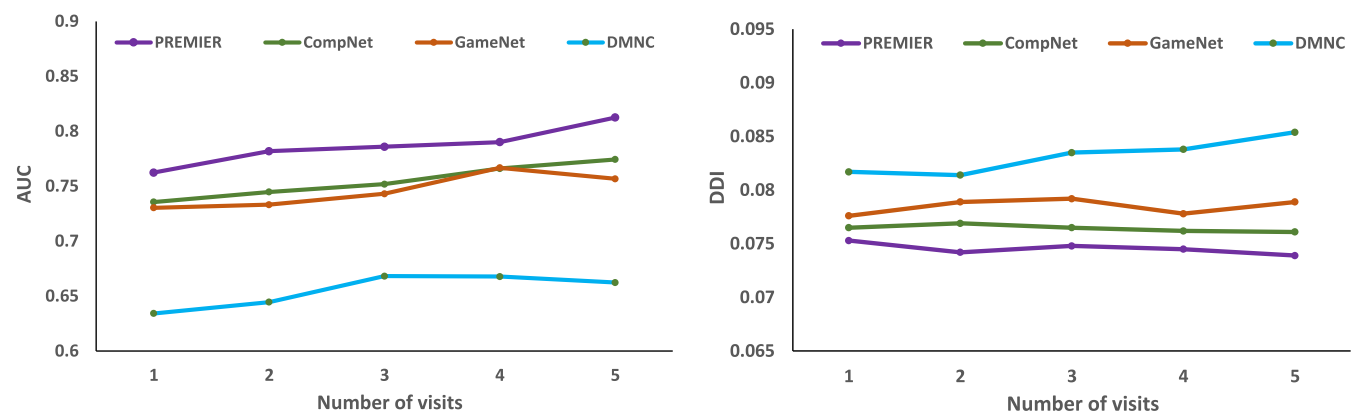

(a) MIMIC-III
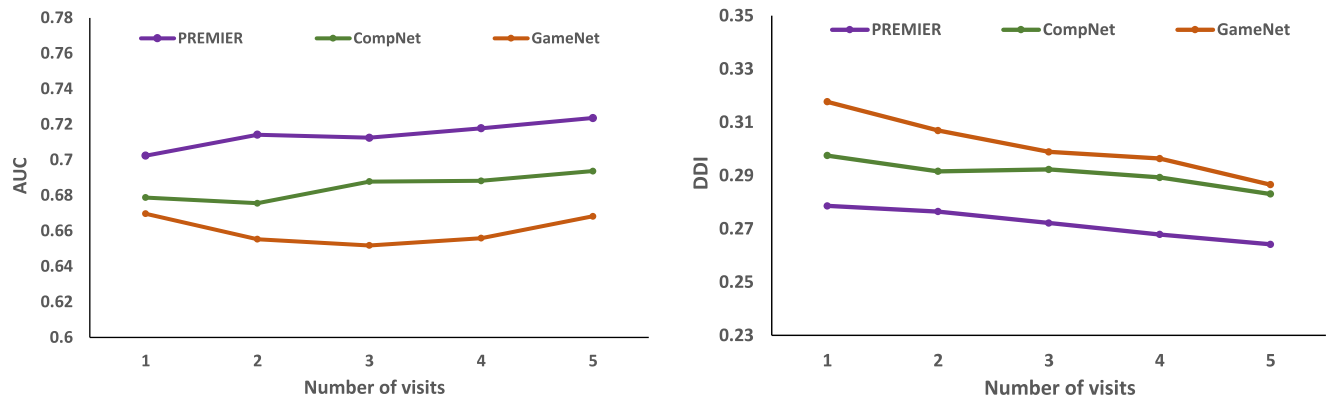

(b) PRIVATE

Fig. 7. Effect of the number of visits on AUC and DDI.

Table 5. Effect of Visit-Aware and Diagnosis/Procedure-Aware Attentions

(a) MIMIC-III

\begin{tabular}{|l|c|c|c|c|c|c|c|}
\hline \multirow{2}{*}{ Methods } & \multicolumn{3}{|c|}{ Accuracy } & $\begin{array}{c}\text { Drug } \\
\text { Interaction }\end{array}$ & \multicolumn{3}{c|}{ Tradeoff } \\
\cline { 2 - 8 } & AUC & F1 & Jaccard & DDI & DScore $_{\text {AUC }}$ & DScore $_{\text {F1 }}$ & DScore $_{\text {Jac }}$ \\
\hline \hline PREMIER $_{\boldsymbol{\alpha}}$ & 0.7464 & 0.6638 & 0.5091 & 0.0760 & 0.8257 & 0.7725 & 0.6564 \\
\hline PREMIER $_{\boldsymbol{\beta}}$ & 0.7556 & 0.6736 & 0.5142 & 0.0762 & 0.8312 & 0.7791 & 0.6606 \\
\hline PREMIER $^{\mathbf{0 . 7 7 9 5}}$ & $\mathbf{0 . 6 8 1 2}$ & $\mathbf{0 . 5 2 6 9}$ & $\mathbf{0 . 0 7 5 0}$ & $\mathbf{0 . 8 4 6 0}$ & $\mathbf{0 . 7 8 4 5}$ & $\mathbf{0 . 6 7 1 3}$ \\
\hline
\end{tabular}

(b) PRIVATE

\begin{tabular}{|l|c|c|c|c|c|c|c|}
\hline \multirow{2}{*}{ Methods } & \multicolumn{3}{|c|}{ Accuracy } & $\begin{array}{c}\text { Drug } \\
\text { Interaction }\end{array}$ & \multicolumn{3}{c|}{ Tradeoff } \\
\cline { 2 - 8 } & AUC & F1 & Jaccard & DDI & DScore $_{\text {AUC }}$ & DScore $_{\text {F1 }}$ & DScore $_{\text {Jac }}$ \\
\hline \hline PREMIER $_{\boldsymbol{\alpha}}$ & 0.6823 & 0.6175 & 0.5100 & 0.2790 & 0.7011 & 0.6652 & 0.5974 \\
\hline PREMIER $_{\boldsymbol{\beta}}$ & 0.6945 & 0.6301 & 0.5212 & 0.2789 & 0.7075 & 0.6725 & 0.6050 \\
\hline PREMIER $^{\mathbf{0 . 7 1 8 9}}$ & $\mathbf{0 . 6 4 0 8}$ & $\mathbf{0 . 5 3 9 8}$ & $\mathbf{0 . 2 7 1 9}$ & $\mathbf{0 . 7 2 3 4}$ & $\mathbf{0 . 6 8 1 6}$ & $\mathbf{0 . 6 1 9 9}$ \\
\hline
\end{tabular}

Performance on rare diseases. We also examine the performance of PREMIER for patients with rare diseases. We use MIMIC-III for this experiment, as PRIVATE is an outpatient dataset with common chronic diseases such as diabetes and hypertension. A disease is considered rare if it affects fewer than 1 in 2,000 people [33]. Given that our dataset has 6,000 patients, we adapt the definition and consider that a disease is rare if the diagnosis code occurs in fewer than 3 patients. 
Table 6. Results for Patients with Rare Diseases

\begin{tabular}{|c|c|c|c|c|c|c|c|}
\hline \multirow{2}{*}{ Methods } & \multicolumn{3}{|c|}{ Accuracy } & $\begin{array}{c}\text { Drug } \\
\text { Interaction }\end{array}$ & \multicolumn{3}{c|}{ Tradeoff } \\
\cline { 2 - 8 } & AUC & F1 & Jaccard & DDI & DScore $_{\text {AUC }}$ & DScore $_{\text {F1 }}$ & DScore Jac $^{\text {DSc }}$ \\
\hline \hline LR & 0.6586 & 0.6204 & 0.4567 & 0.0733 & 0.7699 & 0.7432 & 0.6118 \\
\hline K-frequent & 0.7148 & 0.6214 & 0.4695 & 0.0727 & 0.8073 & 0.7441 & 0.6233 \\
\hline LEAP & 0.6501 & 0.6171 & 0.4464 & $\mathbf{0 . 0 5 8 3}$ & 0.7691 & 0.7456 & 0.6056 \\
\hline DMNC & 0.6147 & 0.5485 & 0.3862 & 0.0629 & 0.7424 & 0.6919 & 0.5469 \\
\hline GameNet & 0.7593 & 0.6873 & 0.5215 & 0.0720 & 0.8352 & 0.7897 & 0.6677 \\
\hline CompNet & 0.7653 & 0.6877 & 0.5327 & 0.0710 & 0.8392 & 0.7903 & 0.6771 \\
\hline PREMIER & $\mathbf{0 . 8 0 4 0}$ & $\mathbf{0 . 7 0 6 4}$ & $\mathbf{0 . 5 5 3 4}$ & 0.0621 & $\mathbf{0 . 8 6 5 8}$ & $\mathbf{0 . 8 0 5 8}$ & $\mathbf{0 . 6 9 6 0}$ \\
\hline
\end{tabular}

Our test dataset has 1,059 patients, and there are 8 patients with rare disease. Table 6 shows that PREMIER continues to perform well for this group of patients compared to the other techniques.

\subsection{Ablation Study}

In this section, we carry out an ablation study to better understand the impact of the various components in PREMIER on its performance. We have the following variants:

(1) PREMIER [diagnosis, procedure]: This variant uses only the diagnosis and procedure codes of all the visits to predict the list of medications. It does not make use of the past prescribed medications and drug interaction information.

(2) PREMIER [diagnosis, procedure, $\boldsymbol{A}_{C}$ ]: This model uses only the diagnosis and procedure codes with only the drug co-occurrences information in the repository. It does not use the medications from the past visits of patients and the information about interacting medications in the DDI database. In other words, patients' previously prescribed medication and drug interaction information is not incorporated in this model.

(3) PREMIER [diagnosis, procedure, $A_{D}$ ]: This model uses only the diagnosis and procedure codes with only the drug interaction information in the repository. It does not use the medications from the past visits of patients and the information about co-occurring medications in the EHR. In other words, patients' previously prescribed medication and drug co-occurrence information is not incorporated in this model.

(4) PREMIER [diagnosis, procedure, $A_{C}, A_{D}$ ]: This model uses only the diagnosis and procedure codes along with drug information in the repository. It does not use the medications from the past visits of patients. In other words, patients' previously prescribed medication information is not incorporated in this model.

(5) PREMIER [diagnosis, procedure, medication]: This model does not have the drug interaction information. In other words, it uses the diagnosis and procedure codes, as well as the medication prescribed, in the past visits of patients.

Table 7(a) and (b) present the results for these variants of PREMIER on MIMIC-III and PRIVATE, respectively. As expected, using only diagnosis and procedure information has the lowest accuracy and DScores with the highest DDI. Incorporating drug co-occurrence and drug interaction information helps reduce the DDI. We observe improvements in all the accuracy measures and DScore when we take into account past medications. Compared to PREMIER [diagnosis, procedure], we see that the DDI is lower for other variants because the past medication information implicitly contains some knowledge of drug co-occurrences and drug interactions. PREMIER [diagnosis, 
Table 7. Results of the Ablation Study

(a) MIMIC-III

\begin{tabular}{|c|c|c|c|c|c|c|c|}
\hline \multirow{2}{*}{ Methods } & \multicolumn{3}{|c|}{ Accuracy } & \multirow{2}{*}{\begin{tabular}{|c|}
$\begin{array}{c}\text { Drug } \\
\text { Interaction }\end{array}$ \\
DDI \\
\end{tabular}} & \multicolumn{3}{|c|}{ Tradeoff } \\
\hline & \begin{tabular}{|l|} 
AUC \\
\end{tabular} & F1 & Jaccard & & DScore $_{\mathrm{AUC}}$ & DScore $_{\mathrm{F} 1}$ & DScore $_{\text {Jac }}$ \\
\hline "PREMIER [diagnosis, procedure] & 0.7456 & 0.6643 & 0.5086 & 0.0810 & 0.8232 & 0.7711 & 0.6548 \\
\hline PREMIER [diagnosis, procedure, $A_{C}$ ] & 0.7465 & 0.6673 & 0.5090 & 0.0785 & 0.8248 & 0.7740 & 0.6557 \\
\hline PREMIER [diagnosis, procedure, $A_{D}$ ] & 0.7461 & 0.6629 & 0.5088 & 0.0776 & 0.8249 & 0.7714 & 0.6558 \\
\hline PREMIER [diagnosis, procedure, $A_{C}, A_{D}$ ] & 0.7473 & 0.6650 & 0.5094 & 0.0781 & 0.8254 & 0.7726 & 0.6562 \\
\hline PREMIER [diagnosis, procedure, medication] & 0.7508 & 0.6701 & 0.5103 & 0.0790 & 0.8272 & 0.7757 & 0.6567 \\
\hline PREMIER & 0.7795 & 0.6812 & 0.5269 & 0.0750 & 0.8460 & 0.7845 & 0.6713 \\
\hline
\end{tabular}

(b) PRIVATE

\begin{tabular}{|c|c|c|c|c|c|c|c|}
\hline \multirow{2}{*}{ Methods } & \multicolumn{3}{|c|}{ Accuracy } & \multirow{2}{*}{\begin{tabular}{|c|}
$\begin{array}{c}\text { Drug } \\
\text { Interaction }\end{array}$ \\
DDI \\
\end{tabular}} & \multicolumn{3}{|c|}{ Tradeoff } \\
\hline & AUC & F1 & Jaccard & & DScore $_{\mathrm{AUC}}$ & DScore $_{\mathrm{F} 1}$ & DScore $_{\text {Jac }}$ \\
\hline PREMIER [diagnosis, procedure] & 0.6745 & 0.6117 & 0.5002 & 0.3078 & 0.6832 & 0.6494 & 0.5807 \\
\hline PREMIER [diagnosis, procedure, $A_{C}$ ] & 0.6812 & 0.6185 & \begin{tabular}{|l|}
0.5106 \\
\end{tabular} & 0.2945 & 0.6931 & 0.6591 & 0.5924 \\
\hline PREMIER [diagnosis, procedure, $A_{D}$ ] & 0.6803 & 0.6164 & \begin{tabular}{|l|}
0.5078 \\
\end{tabular} & 0.2856 & 0.6969 & 0.6617 & 0.5936 \\
\hline PREMIER [diagnosis, procedure, $A_{C}, A_{D}$ ] & 0.6857 & 0.6195 & 0.5128 & 0.2851 & 0.6999 & 0.6637 & 0.5972 \\
\hline PREMIER [diagnosis, procedure, medication] & 0.6926 & 0.6235 & 0.5164 & 0.2983 & 0.6971 & 0.6602 & 0.5949 \\
\hline PREMIER & 0.7189 & 0.6408 & 0.5398 & 0.2719 & 0.7234 & 0.6816 & 0.6199 \\
\hline
\end{tabular}

procedure, $A_{D}$ ] and PREMIER [diagnosis, procedure, $A_{C}$ ] do not perform as well compared to using both drug co-occurrence and drug interaction information.

\subsection{PREMIER with Transformer/BERT}

In this set of experiments, we replace the neural attention models in stage 1 of PREMIER (see Figure 3) with two popular sequence modeling techniques: Transformer [41] and BERT [7].

Similar to Vaswani et al. [41], Transformer/BERT takes as inputs the concatenation of a sinusoidal positional encoding and a linear encoding. The outputs of the Transformer/BERT last encoder layer over every timestep until $T$ are summed to obtain the response vectors, $\boldsymbol{r}^{d}$ and $\boldsymbol{r}^{p}$. The context vector, $c$, is obtained using Equation (4), as described in Section 3.1. Note that the BERT model has been pre-trained for the medical domain based on the techniques in the work of Shang et al. [29]. The hidden dimension and dimension of the feed-forward network are set to 256 for both PREMIER with Transformer/BERT on both datasets. For MIMIC-III, PREMIER with Transformer consists of four stacked transformer encoder units with two attention heads for each layer, whereas PREMIER with BERT consists of two stacked transformer encoder units with two attention heads for each layer. For PRIVATE, PREMIER with Transformer consists of six stacked transformer encoder units with four attention heads for each layer, whereas PREMIER with BERT consists of two stacked transformer encoder units with two attention heads for each layer. Training is done using Adam [18] with a learning rate of 0.0001 for both datasets.

Table 8 shows the results on MIMIC-III and PRIVATE. We see that the PREMIER with BERT gives the best performance. However, the presence of a non-linear layer in BERT makes it difficult to compute the justification scores. Since providing an explanation on why a specific medication is suggested is an important consideration for clinical acceptance of a recommendation system, we have chosen to adopt the neural attention based sequential modeling in PREMIER.

\subsection{Case Study}

We present two use cases to demonstrate how PREMIER provides justification for the recommended medications. 
Table 8. Results of PREMIER with Transformer/BERT

(a) MIMIC-III

\begin{tabular}{|c|c|c|c|c|c|c|c|}
\hline \multirow{2}{*}{ Methods } & \multicolumn{3}{|c|}{ Accuracy } & $\begin{array}{c}\text { Drug } \\
\text { Interaction }\end{array}$ & \multicolumn{3}{c|}{ Tradeoff } \\
\cline { 2 - 8 } & AUC & F1 & Jaccard & DDI & DScore $_{\text {AUC }}$ & DScore $_{\text {F1 }}$ & DScore $_{\text {Jac }}$ \\
\hline \hline PREMIER & 0.7795 & 0.6812 & 0.5269 & 0.0750 & 0.8460 & 0.7845 & 0.6713 \\
\hline PREMIER with Transformer & 0.8018 & 0.7053 & 0.5487 & 0.0752 & 0.8589 & 0.8002 & 0.6887 \\
\hline PREMIER with BERT & $\mathbf{0 . 8 2 5 0}$ & $\mathbf{0 . 7 1 9 2}$ & $\mathbf{0 . 5 6 4 3}$ & $\mathbf{0 . 0 7 4 8}$ & $\mathbf{0 . 8 7 2 2}$ & $\mathbf{0 . 8 0 9 2}$ & $\mathbf{0 . 7 0 1 0}$ \\
\hline
\end{tabular}

(b) PRIVATE

\begin{tabular}{|c|c|c|c|c|c|c|c|}
\hline \multirow{2}{*}{ Methods } & \multicolumn{3}{|c|}{ Accuracy } & \multirow{2}{*}{\begin{tabular}{|c|}
$\begin{array}{c}\text { Drug } \\
\text { Interaction }\end{array}$ \\
DDI \\
\end{tabular}} & \multicolumn{3}{|c|}{ Tradeoff } \\
\hline & AUC & F1 & Jaccard & & DScore $_{\text {AUC }}$ & DScore $_{\mathrm{F} 1}$ & DScore Jac $_{\text {Ja }}$ \\
\hline 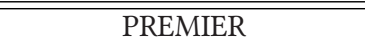 & 0.7189 & 0.6408 & 0.5398 & 0.2719 &  & 0.6816 & 0.6199 \\
\hline 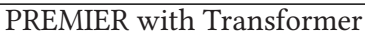 & 0.7351 & 0.6545 & 0.5503 & 0.2622 & 0.7364 & 0.6936 & 0.6304 \\
\hline $\begin{array}{l}\text { PREMIER with BERT } \\
\end{array}$ & 0.7527 & 0.6801 & 0.5682 & 0.2606 & 0.7459 & 0.7085 & 0.6425 \\
\hline
\end{tabular}

Patient A. This patient has been diagnosed with malignant neoplasm of the liver and druginduced delirium, and subsequently went on to have liver transplant surgery. The patient returned for a second visit and was diagnosed with septic shock with severe sepsis, a potentially life-threatening condition caused by the body's response to an infection caused by bacteria. ${ }^{2}$

We analyze the medications recommended to Patient A and observe that the diagnosis contributes nearly $49 \%$ to the list of medications recommended, procedures contribute close to $39 \%$, and past medications contribute to the remaining $12 \%$. Table 9 gives a sample of the prescribed medications, recommended medications, and the corresponding justifications based on the top two contribution scores obtained from PREMIER. We see that the justifications for the medications in rows 1 through 3 are primarily due to the diagnosis of sepsis. The first two medications, ciprofloxacin and piperacillin, belong to the class of intestinal anti-infectives and beta-lactam antibacterials, respectively. The justifications make sense, as these medications are known to treat sepsis [6]. The second highest contributor in row 2 is the liver transplant procedure, which Patient A has undergone previously. This makes sense, as liver transplant is known to increase the risk of sepsis. The third medication is zinc sulfate, a mineral supplement that is often prescribed to prevent infections or recurrence of sepsis [10]. Row 4 shows that calcium is recommended with the justification of septic shock, another diagnosis for Patient A. This is reasonable, as calcium is typically administered for resuscitation after septic shock [3]. The medication acetaminophen in row 5 is an analgesic and anti-pyretic, and the justification shows it is mainly due to the diagnosis of delirium. Delirium is a serious disturbance in mental abilities that results in confused thinking and reduced awareness of the environment. ${ }^{3}$ Since delirium often stems from pain and fever, analgesics and anti-pyretics are administered to reduce the pain and body temperature to reduce the severity of delirium [9]. We see that the justifications for the medications recommended are appropriate and aligned with clinical practices.

Patient B. This patient has been diagnosed with coronary atherosclerosis and has undergone a surgery involving the bypass of three coronary arteries. The patient is subsequently diagnosed with severe tracheal disorder and is admitted for a procedure of invasive mechanical ventilation.

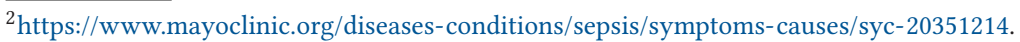

${ }^{3}$ https://www.mayoclinic.org/diseases-conditions/delirium/symptoms-causes/syc- 20371386.
} 
Table 9. Sample Medications Recommended to Patient A and Their Justifications

\begin{tabular}{|l|l|l|l|}
\hline & Prescribed Medications & Recommended Medications & \multicolumn{1}{|c|}{ Justification $^{+}$} \\
\hline 1 & Ciprofloxacin & Intestinal anti-infectives & Sepsis (90.36), Septic shock (4.60) \\
\hline 2 & Piperacillin and tazobactam & Beta-lactam anti-bacterials & Sepsis (39.64), Liver transplant (20.97) \\
\hline 3 & Zinc sulfate & Mineral supplements & Sepsis (31.48), Lacrimal gland operated (26.19) \\
\hline 4 & Calcium gluconate & Calcium & Septic shock (30.63), Omeprazole (20.56) \\
\hline 5 & Acetaminophen & Analgesics and anti-pyretics & Delirium (48.52), Opioids (15.36) \\
\hline
\end{tabular}

${ }^{+}$Blue indicates diagnosis, orange indicates procedures, and green indicates past medications.

Table 10. Sample Medications Recommended to Patient B and Their Justifications

\begin{tabular}{|l|l|l|l|}
\hline & Prescribed Medications & \multicolumn{1}{|c|}{ Recommended Medications } & \multicolumn{1}{c|}{ Justification $^{+}$} \\
\hline 1 & Captopril & Vasodilators & Coronary bypass (71.34), Atrial fibrillation (19.77) \\
\hline 2 & Acebutolol & Beta-blocking agents & Coronary bypass (89.67), Anemia (2.46) \\
\hline 3 & Cisatracurium besylate & Peripherally acting muscle relaxants & Mechanical ventilation (90.05), Tracheomalacia (7.55) \\
\hline 4 & Diazepam & Hypnotics and sedatives & Mechanical ventilation (77.09), Coronary bypass (20.00) \\
\hline 5 & Magnesium sulfate & Mineral supplements & Mechanical ventilation (57.20), Anemia (30.65) \\
\hline
\end{tabular}

${ }^{+}$Blue indicates diagnosis, orange indicates procedures, and green indicates past medications.

Table 10 shows the prescribed medications, recommended medications, and their corresponding justifications, which are mainly related to the procedure of coronary bypass surgery. Row 1 is a vasodilator to dilate blood vessels and is used to facilitate the flow of blood to reduce the pressure on heart after surgery involving coronaries [17]. Further, the second highest contributor, atrial fibrillation, in row 1 also makes sense, as it leads to heart strokes, which eventually require bypass surgery. Row 2 is a beta-blocking agent to help lower blood pressure, thus preventing complications after a bypass surgery. We observe that a muscle relaxant (row 3 ) is recommended by PREMIER, and the justification given is the mechanical ventilation procedure. This aligns with the fact that muscle relaxants help facilitate the intubation or ventilation procedure [31]. The medication in row 4 is diazepam, which belongs to the class of hypnotics and sedatives and is used to stabilize patients under ventilation [11]. Since this patient is under invasive mechanical ventilation, mineral supplements (row 5) are also recommended to replenish the physiological stability.

The preceding two cases studies indicate that PREMIER is able to provide sensible justifications.

\subsection{Manual Assessment of Justifications}

Finally, we randomly pick 10 patients from the MIMIC-III dataset and generate PREMIER's recommended medications with their justifications for the first two visits. The top justification for each recommended medication is shown to a clinician. Out of a total of 72 justifications, the clinician assessed 68 as correct and 4 as incorrect. Table 11 shows a sample of the results. We take a closer look at the justifications deemed incorrect by the clinician.

For Patient 1, the justification provided by PREMIER for recommending vitamin B12 and folic acid is congestive heart failure. Although this medication is generally prescribed for alleviating anemia, we find that congestive heart failure often induces anemia [39]. This comorbidity occurs frequently in our datasets, causing PREMIER to give the wrong justification. For Patient 4 , the justification for recommending anti-arrhythmics is acute diastolic heart failure. This medication is typically prescribed for atrial fibrillation, and patients with a heart failure condition tend to develop atrial fibrillation [23], resulting in PREMIER being mistaken about the actual reason for the medication. Patient 6 is recommended drugs for constipation. Although the justification is wrongly given as acute kidney failure, studies have shown that constipation is highly prevalent in patients with kidney failure [15]. Patient 10 is recommended mineral supplements, and the reason given is because the patient is under mechanical ventilation. Although this is not correct, the work 
Table 11. Sample Assessment of Justifications

\begin{tabular}{|c|c|c|c|}
\hline Patient & Recommendations & Justification $^{+}$ & Assessment \\
\hline \multirow[t]{3}{*}{1} & Analgesics and anti-pyretics & Urinary tract infection & Correct \\
\hline & Anti-varicose therapy & $\begin{array}{l}\text { Endovascular replacement } \\
\text { of aortic valve }\end{array}$ & Correct \\
\hline & Vitamin B12 and folic acid & Congestive heart failure & Incorrect \\
\hline \multirow[t]{3}{*}{2} & Beta-blocking agents & Chronic diastolic heart failure & Correct \\
\hline & Analgesics and anti-pyretics & Pain in joint and shoulder region & Correct \\
\hline & Anti-thrombotic agents & Acute myocardial infarction & Correct \\
\hline \multirow[t]{3}{*}{3} & Hypnotics and sedatives & $\begin{array}{l}\text { Continuous invasive mechanical } \\
\text { ventilation }\end{array}$ & Correct \\
\hline & Anti-varicose therapy & Chronic diastolic heart failure & Correct \\
\hline & Beta-lactum anti-bacterials & Sepsis & Correct \\
\hline \multirow[t]{3}{*}{4} & Beta-blocking agents & Atrial fibrillation & Correct \\
\hline & Anti-bacterials & Severe sepsis & Correct \\
\hline & Anti-arrhythmics & Acute diastolic heart failure & Incorrect \\
\hline \multirow[t]{3}{*}{5} & Anti-bacterials & Severe sepsis & Correct \\
\hline & Anti-depressants & Dysthymic disorder & Correct \\
\hline & Drugs for addictive disorders & Alcohol abuse & Correct \\
\hline \multirow[t]{3}{*}{6} & Potassium-sparing agents & Hyperaldosteronism & Correct \\
\hline & ACE inhibitors & Congestive heart failure & Correct \\
\hline & Drugs for constipation & Acute kidney failure & Incorrect \\
\hline \multirow[t]{3}{*}{7} & Anti-varicose therapy & Portal hypertension & Correct \\
\hline & Beta-blocking agents & Bypass of coronary arteries & Correct \\
\hline & Potassium-sparing agents & Acute kidney failure & Correct \\
\hline \multirow[t]{2}{*}{8} & ACE inhibitors & Unspecified essential hypertension & Correct \\
\hline & Anti-cholinergic agents & Paranoid type schizophrenia & Correct \\
\hline \multirow[t]{3}{*}{9} & Anti-inflamatory and anti-rheumatic & Liver transplant & Correct \\
\hline & Mineral supplements & Anemia & Correct \\
\hline & Intestinal anti-infectives & Sepsis & Correct \\
\hline \multirow[t]{4}{*}{10} & Beta-blocking agents & Chronic diastolic heart failure & Correct \\
\hline & Analgesics and anti-pyretics & Fever & Correct \\
\hline & Vasodilators & Bypass of coronary arteries & Correct \\
\hline & Mineral supplements & Mechanical ventilation & Incorrect \\
\hline
\end{tabular}

${ }^{+}$Blue indicates the diagnosis, and orange indicates procedures.

of Silver et al. [32] shows that patients under mechanical ventilation usually suffer from anemia and hence are prescribed mineral supplements. The preceding analysis reveals that PREMIER does not provide totally irrelevant justifications, but the recommended medication may reveal the cause or precursor of the medical condition.

\section{CONCLUSION AND FUTURE WORK}

In this work, we have described an end-to-end system called PREMIER that takes into consideration information from past and current visits along with drug information to make accurate recommendations that minimize adverse drug interactions. The system works in two stages and jointly models the sequential dependencies in past visits and graphical representations of drug co-occurrence and interaction information to derive the recommendations and their justifications. Experimental results on both public and private EHR datasets demonstrate the ability of PREMIER to outperform existing solutions in attaining the best tradeoff. Case studies and clinician assess- 
ment also show that the justifications are appropriate and help patients and clinicians understand why the medications are recommended. Future work includes incorporating lab reports and medical notes, which are rich sources of information for additional insights such as patient allergies.

\section{REFERENCES}

[1] European Pharmaceutical Marketing Research Association. 2013. EphMRA Anatomical Classification Guidelines. EphMRA, Bromley, UK.

[2] Jacek M. Bajor and Thomas A. Lasko. 2017. Predicting medications from diagnostic codes with recurrent neural networks. In Proceedings of ICLR.

[3] Kenneth W. Burchard, H. Hank Simms, Ann Robinson, Ronald DiAmico, and Donald S. Gann. 1992. Hypocalcemia during sepsis: Relationship to resuscitation and hemodynamics. Archives of Surgery 127, 3 (1992), 265-272.

[4] Zhuo Chen, Kyle Marple, Elmer Salazar, Gopal Gupta, and Lakshman Tamil. 2016. A physician advisory system for chronic heart failure management based on knowledge patterns. Theory and Practice of Logic Programming 16, 5-6 (2016), 604-618.

[5] Edward Choi, Mohammad Taha Bahadori, Jimeng Sun, Joshua Kulas, Andy Schuetz, and Walter Stewart. 2016. Retain: An interpretable predictive model for healthcare using reverse time attention mechanism. In Proceedings of NIPS. 3504-3512.

[6] Yann-Erick Claessens and Jean-François Dhainaut. 2007. Diagnosis and treatment of severe sepsis. Critical Care 11, 5 (2007), S2.

[7] Jacob Devlin, Ming-Wei Chang, Kenton Lee, and Kristina Toutanova. 2018. Bert: Pre-training of deep bidirectional transformers for language understanding. arXiv preprint arXiv:1810.04805 (2018).

[8] Ronald Aylmer Fisher. 1992. Statistical methods for research workers. In Breakthroughs in Statistics. Springer, 66-70.

[9] S. Fosnight. 2011. Delirium in the elderly. In Pharmacotherapy Self-Assessment Program, Geriatrics, C. Chant, K. H. Chessman, S. W. Finks, B. A. Hemstreet, and A. L. Hume (Eds). American College of Clinical Pharmacy, 73-95.

[10] Salah Gariballa. 2005. Vitamin and mineral supplements for preventing infections in older people. BMJ 331, 7512 (2005), 304-305.

[11] Mary Jo Grap, Cindy L. Munro, Paul A. Wetzel, Al M. Best, Jessica M. Ketchum, V. Anne Hamilton, Nyimas Y. Arief, Rita Pickler, and Curtis N. Sessler. 2012. Sedation in adults receiving mechanical ventilation: Physiological and comfort outcomes. American fournal of Critical Care 21, 3 (2012), e53-e64.

[12] Meredith Gunlicks-Stoessel, Laura Mufson, Ana Westervelt, Daniel Almirall, and Susan Murphy. 2016. A pilot SMART for developing an adaptive treatment strategy for adolescent depression. fournal of Clinical Child \& Adolescent Psychology 45, 4 (2016), 480-494.

[13] Will Hamilton, Zhitao Ying, and Jure Leskovec. 2017. Inductive representation learning on large graphs. In Proceedings of NIPS. 1024-1034.

[14] Anahita Hosseini, Ting Chen, Wenjun Wu, Yizhou Sun, and Majid Sarrafzadeh. 2018. HeteroMed: Heterogeneous information network for medical diagnosis. In Proceedings of CIKM. 763-772.

[15] Ryota Ikee, Kazuhiro Yano, and Tomomi Tsuru. 2019. Constipation in chronic kidney disease: It is time to reconsider. Renal Replacement Therapy 5, 1 (2019), 1-10.

[16] Alistair E. W. Johnson, Tom J. Pollard, Lu Shen, H. Lehman Li-wei, Mengling Feng, Mohammad Ghassemi, Benjamin Moody, Peter Szolovits, Leo Anthony Celi, and Roger G. Mark. 2016. MIMIC-III, a freely accessible critical care database. Scientific Data 3 (2016), 160035.

[17] Joel A. Kaplan. 1981. Peripheral vasodilators in open heart surgery. Cleveland Clinic Quarterly 48, 1 (1981), 174.

[18] Diederik P. Kingma and Jimmy Ba. 2015. Adam: A method for stochastic optimization. In Proceedings of ICLR.

[19] Thomas N. Kipf and Max Welling. 2017. Semi-supervised classification with graph convolutional networks. In IProceedings of ICLR.

[20] Himabindu Lakkaraju and Cynthia Rudin. 2017. Learning cost-effective and interpretable treatment regimes. In AISTATS (Proceedings of Machine Learning Research), Aarti Singh and Jerry Zhu (Eds.), Vol. 54. PMLR, 166-175.

[21] Hung Le, Truyen Tran, and Svetha Venkatesh. 2018. Dual memory neural computer for asynchronous two-view sequential learning. In Proceedings of SIGKDD. ACM, New York, NY, 1637-1645.

[22] Oscar Luaces, Jorge Díez, José Barranquero, Juan José del Coz, and Antonio Bahamonde. 2012. Binary relevance efficacy for multilabel classification. Progress in Artificial Intelligence 1, 4 (2012), 303-313.

[23] Steven A. Lubitz, Emelia J. Benjamin, and Patrick T. Ellinor. 2010. Atrial fibrillation in congestive heart failure. Heart Failure Clinics 6, 2 (2010), 187-200.

[24] Fenglong Ma, Quanzeng You, Houping Xiao, Radha Chitta, Jing Zhou, and Jing Gao. 2018. KAME: Knowledge-based attention model for diagnosis prediction in healthcare. In Proceedings of CIKM. 743-752. 
[25] P. Nguyen, T. Tran, N. Wickramasinghe, and S. Venkatesh. 2017. Deepr: A convolutional net for medical records. IEEE Journal of Biomedical and Health Informatics 21, 1 (Jan. 2017), 22-30.

[26] Jingchao Ni, Hongliang Fei, Wei Fan, and Xiang Zhang. 2017. Cross-network clustering and cluster ranking for medical diagnosis. In Proceedings of ICDE. IEEE, Los Alamitos, CA, 163-166.

[27] Maria Panagioti, Jonathan Stokes, Aneez Esmail, Peter Coventry, Sudeh Cheraghi-Sohi, Rahul Alam, and Peter Bower. 2015. Multimorbidity and patient safety incidents in primary care: A systematic review and meta-analysis. PLoS One 10, 8 (2015), e0135947.

[28] Michael Schlichtkrull, Thomas N. Kipf, Peter Bloem, Rianne Van Den Berg, Ivan Titov, and Max Welling. 2018. Modeling relational data with graph convolutional networks. In The Semantic Web. Lecture Notes in Computer Science, Vol. 10843. Springer, 593-607.

[29] Junyuan Shang, Tengfei Ma, Cao Xiao, and Jimeng Sun. 2019. Pre-training of graph augmented transformers for medication recommendation. In Proceedings of IfCAI. 5953-5959. https://doi.org/10.24963/ijcai.2019/825

[30] Junyuan Shang, Cao Xiao, Tengfei Ma, Hongyan Li, and Jimeng Sun. 2018. GAMENet: Graph augmented memory networks for recommending medication combination. In Proceedings of AAAI.

[31] Michael D. Sharpe. 1992. The use of muscle relaxants in the intensive care unit. Canadian fournal of Anaesthesia 39, 9 (1992), 949-962.

[32] Michael R. Silver. 2005. Anemia in the long-term ventilator-dependent patient with respiratory failure. Chest 128,5 (2005), 568S-575S

[33] Simone Baldovino, Domenica Taruscio, and Dario Roccatello. 2016. Rare diseases in Europe: From a wide to a local perspective. Israel Medical Association fournal 18, 6 (2016), 359-363.

[34] Vergil N. Slee. 1978. The International classification of diseases: Ninth revision (ICD-9). Annals of Internal Medicine 88, 3 (1978), 424-426.

[35] Illés Solt and Domonkos Tikk. 2009. Yet another rule-based approach for extracting medication information from discharge summaries. In Proceedings of the i2b2 Workshop.

[36] Sainbayar Sukhbaatar, Arthur Szlam, Jason Weston, and Rob Fergus. 2015. End-to-end memory networks. In Proceedings of NIPS. 2440-2448.

[37] Leilei Sun, Chuanren Liu, Chonghui Guo, Hui Xiong, and Yanming Xie. 2016. Data-driven automatic treatment regimen development and recommendation. In Proceedings of SIGKDD. ACM, New York, NY, 1865-1874.

[38] Qingxiong Tan, Andy Jinhua Ma, Mang Ye, Baoyao Yang, Huiqi Deng, Vincent Wai-Sun Wong, Yee-Kit Tse, et al. 2019. UA-CRNN: Uncertainty-aware convolutional recurrent neural network for mortality risk prediction. In Proceedings of CIKM. 109-118.

[39] Yi-Da Tang and Stuart D. Katz. 2006. Anemia in chronic heart failure: Prevalence, etiology, clinical correlates, and treatment options. Circulation 113, 20 (2006), 2454-2461.

[40] Nicholas P. Tatonetti, P. Ye Patrick, Roxana Daneshjou, and Russ B. Altman. 2012. Data-driven prediction of drug effects and interactions. Science Translational Medicine 4, 125 (2012), 125ra31-125ra31.

[41] Ashish Vaswani, Noam Shazeer, Niki Parmar, Jakob Uszkoreit, Llion Jones, Aidan N. Gomez, Łukasz Kaiser, and Illia Polosukhin. 2017. Attention is all you need. In Proceedings of NIPS. 5998-6008.

[42] Petar Veličković, Guillem Cucurull, Arantxa Casanova, Adriana Romero, Pietro Liò, and Yoshua Bengio. 2018. Graph attention networks. In Proceedings of ICLR.

[43] Lu Wang, Wei Zhang, Xiaofeng He, and Hongyuan Zha. 2018. Personalized prescription for comorbidity. In Proceedings of DASFAA. 3-19.

[44] Meng Wang, Mengyue Liu, Jun Liu, Sen Wang, Guodong Long, and Buyue Qian. 2017. Safe medicine recommendation via medical knowledge graph embedding. CoRR abs/1710.05980 (2017).

[45] Shanshan Wang, Pengjie Ren, Zhumin Chen, Zhaochun Ren, Jun Ma, and Maarten de Rijke. 2019. Order-free medicine combination prediction with graph convolutional reinforcement learning. In Proceedings of CIKM 2019.

[46] Shangsheng Xie and Mingming Lu. 2019. Interpreting and understanding graph convolutional neural network using gradient-based attribution methods. arXiv preprint arXiv:1903.03768 (2019).

[47] Yutao Zhang, Robert Chen, Jie Tang, Walter F. Stewart, and Jimeng Sun. 2017. LEAP: Learning to prescribe effective and safe treatment combinations for multimorbidity. In Proceedings of SIGKDD. ACM, New York, NY, 1315-1324.

Received November 2020; revised September 2021; accepted September 2021 